\title{
Cyclin Dependent Kinase Inhibitors as Drug Targets for Cancer-A Review
}

\author{
M Krishnaveni* \\ Department of Biochemistry, Periyar University, India \\ *Corresponding author: M Krishnaveni, Assistant Professor, Department of Biochemistry, Periyar University, Salem-636 011, India \\ Submission: 監September 18, 2017; Published: 酱 October 11, 2017
}

\section{Introduction}

Cyclin dependent kinases are proline directed serine / threonine protein kinases comprising of catalytic CDK subunit and active cyclin subunit. Till now around 25 different cyclins and 13 different cyclin dependent kinases are reported but only 7 CDK's play a major role in the progression of cell cycle. Usually throughout cell cycle, cyclin levels vary and CDK remains constant. Complete activation of cyclin dependent kinases takes place by the phosphorylation of threonine residue close to kinase active site. Cyclin dependent kinases phosphorylate various substrates that are required for the progression of cell cycle. The eukaryotic cell growth and cell division are regulated at late G1, G2/M, metaphase to anaphase transition (check points). The first two check points are controlled by cyclin dependent kinases and are specific for each phase like CDK 4, 6 controls late G1 and S phase by CDK2 and CDK 1 , while G2/M and last by anaphase promoting complex. Cyclin D interaction with CDK 4 regulates $S$ phase initially, apart from this CDK 4/6 regulation at transcriptional level controls late G1 phase. Loss of cell cycle control, hyper-activation of cyclin dependent kinases due to mutation causes cancer. So, inhibitions of cyclin dependent kinases are targets in cancer therapy. The inhibitors of cyclin dependent kinases are ATP-competitive, ATP-noncompetitive (including some small mimetic peptides for p21, p27 and p57), Allosteric inhibitors. Let us have a look on some of the inhibitors in this review.

\section{Structure of Cyclin Dependent Kinases}

The CDK was first identified in yeast and frog, in fungi 8 CDK's and around 15 cyclins were present, while flies, echinodermata contain 11 CDK's and 14 cyclins and human beings have 20 CDK's and 29 cyclins [1]. CDKs are two-lobed structure composed of betasheets at its amino-terminal and $\alpha$-helices in its carboxy-terminal, and in-between, the active site is located. The N-lobe has glycinerich inhibitory element (G-loop), unique major helix - the C-helix having PSTAIRE sequence in Cdk1. The C-lobe's activation segment spans from DFG motif (D145 in Cdk2; EMBL: AK291941) to APE motif (E172 in Cdk2) and includes the phosphorylation-sensitive (T160 in Cdk2) residue in the so-called T-loop. The glycine-rich region (G-loop) in the N-lobe acts as an extra regulatory region as it have Thr14 and Tyr15 in Cdk2. Phosphorylation of Thr14, Tyr15 residues by Wee1 and Myt1 kinases inhibits proteins preventing cell-cycle progression when a cell is exposed to DNA damage. Phosphate removal by phosphates of the Cdc25 family activates CDKs, cell-cycle progression [2,3].

\section{Types of CDK Inhibitors}

\section{ATP competitive CDK inhibitors}

Most of the CDK inhibitors belong to ATP competitive type and are found to act by interacting with catalytic ATP site of CDK's. Examples for this type of inhibitors are flavones, indole, purine, thiozole, and pyrazoles. The first generation CDK inhibitors that enter into clinical trial of anticancer therapy are flavopiridol and roscovitine. Flavopiridol is a naturally occurring alkaloid, low in molecular weight. Flavopiridol inhibit CDK 1, 4, 6, 7, 9 and roscovitine inhibits CDK 1, 2, 7, 9 but CDK4/6 inhibition was poor. The flavopiridol inhibitory effect on CDK's are competed away by the addition of excess ATP but it was not the same with CDK9/Cyclin T. Cell cycle arrest may takes place either at G1-S or at G2-M transition with flavopiridol. G1-S arrest takes place partly by the inactivation of $\mathrm{CDK} 2 / 4 / 6$ and at the same time flavopiridol also inhibits expression of cyclin D1 thereby inhibits activation of cyclin dependent kinase needed for G1 phase by CDK activation kinase, which also simultaneously causes G2-M arrest. Cyclin D1 forms a basic connection among mitogen and cell cycle, causing mainly diseases like cancer. Flavopiridol also found to inhibit glycogen synthase kinase $3 \beta$. Flavopiridol along with paclitaxel was hopeful in human clinical trials. Similar to flavopiridol, deschloroflavopiridol exhibit cytotoxic effect over several tumor cell lines [4-6]. 6-dimethyl analog inhibited CDK1, 2, 5, MAPK leaving CDK4/6. Purvalanol B was highly effective for CDK2/cyclin A than olomoucine. 6-dimethyl aminopurine was the first CDK1 inhibitor [7], next was olomoucine a purine analog act on CDK1, 2, 5 and not on CDK4/6. The presence of bulky substituents at position 2, 9 of roscovitine showed increased activity [8]. Staurosporine, from Streptomyces inhibits PKC initially and later found that; 
it also inhibits CDK1/cyclin B complexes. In addition to this, Staurosporine derivatives like UCN -01, K252C, CGP41251 are good anti-tumor agents on few cancer cells. UCN-01 reduces $\mathrm{Rb}$ protein phosphorylation in order to block G1/S transition and act indirectly on CDK's at lower concentration but act specifically at higher concentrations. It also act on various kinases including PKC, CDK, Wee1, PDK1 and the anticancer effect was partly attributed by the Wee1 inhibition, causing CDK1 activation followed by premature entry into mitosis, apoptosis. It also inhibit PDK 1 as it is involved in the activation of AKT by phophorylating it, leading to cancer progression $[9,10]$. Butyrolactone I from Aspergillus terreus var. Africans inhibited CDK 1/2 [11]. Hymenialdisine, fascaplysine from marine sponge inhibits cyclin-dependent kinases [12,13]. Meriolins a synthetic hybrid derived from two natural kinase inhibitors from marine invertebrates, meridianins from south atlantic ascidian Aplidium meridianum, variolins extracted from the antartic sponge Kirkpatrickia variolosa. Meriolins (3-2-aminopyrimidine indole) bind strongly with CDK when compared to Variolin B [1417]. Since, toxic effect observed with ATP competitive inhibitor (first generation) under clinical trial were found to be effective when given along with anticancer drugs doxorubicin, cisplatin, HER2 inhibitors or HDAC inhibitor vorinostat, second generation inhibitors (P276-00) developed was found to act against multiple myeloma, melanoma, mantle cell lymphoma expressing increased cyclin D and are more specific $[18,19]$. R03306 a thiazolinone, explains the mitotic functions of human CDK1. SNS-032 thiazole inhibitor acts on CDK2, 7, 9. More recently, it was shown to target AML cells and to be highly synergistic with cytarabine [20-23].

\section{ATP Non-competitive CDK inhibitors}

These inhibitors were not tested through clinical trials, it never compete with ATP for its entry. Very few come under this class of inhibitors. They are small molecule inhibitors (3 aminothio acridinone) and small peptide inhibitors (developed on the basis of similarity with endogenous CDKs inhibitors such as p21, p27, p57). Small peptide inhibitors have common eight residue motif through which it regulate the CDK2/Cyclin activity. Kubo et al. [24-26] identified 3-aminothio acridone a small molecule inhibits CDK4cyclin D1 in patients with tumor carrying p16 defects. Diccianni et al showed its anticancer role in $\mathrm{T}$ cell acute lymphocytic leukemia. SU9516 plays a dual function of both acting as an ATP competitive and ATP non competitive inhibitors. By acting as an ATP competitive inhibitor it inhibits CDK2-cyclin A, CDK2-cyclin E and CDK1-cyclin B1complex, while inhibits CDK4-cyclin D1 alone by functioning as an ATP non competitive inhibitor which was less potent [27]. Compound 1 inhibits CDK 4 and phosphorylation of $\mathrm{Rb}$ in MDA-MB-435 cancer cells and further for cyclin D1 inhibition [28]. Aminoacid heptapeptide NBI1 a small peptide inhibit CDK2cyclin A complex mainly the cyclin A, CDK1 cyclin B1, CDK6-cyclin D3 and moderate inhibitory effect on CDK2-Cyclin E, PKC II , GSK3 $\beta$. In addition to this a cell permeable NBI1 analog induces apoptosis in HCT116, HT29, T98G and A2780 cells [29]. Among the different CDK2-cyclin A inhibitors like p107, pRb2/p130, E2F1, a short sequence containing 39 amino acids, belonging to the spacer domain of RB2/p130 (Spa310), is required for the inhibition of CDK2-cyclin A complex. Spa 310 suppress tumor induced by A549 cells in nude mice [30] and could also be used as antitumor drug. C4 peptide from 285-306 residues in the a5 helix amino acid chain of cyclin A inhibit CDK2-cyclin A complex and also tumor progression in MDA-MB-231 cells. Another variant of C4 combined with Tat protein showed higher inhibitory activity over breast cancer cells. The peptides derived from $\mathrm{p} 21$ called HAKRRLIF mimic the function of p21 by inhibiting G1 phase CDK- cyclin complex especially CDK2cyclin A2 and CDK4-cyclin D1. Peptide from p107 called SAKRRLFG inhibits CDK2-cyclin A2 and CDK4-cyclin D1 complex [31]. CYC 103 inhibit CDK2-cyclin A complex in an unknown manner.

\section{Allosteric inhibitors}

Till now no inhibitors of this type were identified. Schonbrunn team discovered an allosteric site for CDK2 inorder to alter the interaction with cyclin. The fluorophore 1-Anilino Nathalene 8Sulphonic acid was used to know the allosteric site within CDK2. The link between fluorophore and CDK2 alters PSTAIRE helix confirmation thus making the cyclin binding impossible [32]. 5,7-dihydroxyflavone showed therapeutic properties on cancers. A chrysin derivative named compound 69407, an efficient ATPnoncompetitive inhibitor of CDK2, CDK4 binds to an allosteric site of cyclin dependent kinases, reduces cell cycle progression in EGFstimulated cells during G1 phase, inhibited G1/S transition, causing retinoblastoma phosphorylation by CDK4/6, CDK2 [33,34].

\section{Conclusion}

Cyclin dependent kinases are the targets for anticancer therapy among the CDK involved in cell cycle CDK4/6 are prime importance as they regulate G1 phase and it is the decision making step. But as far as today only very few first generation inhibitors were used commercially and rest of the compounds under this group were under clinical trial. Inhibitor that were able to inhibit specifically will be of much useful than broadly inhibiting cyclin dependent kinases. Hence, it is essential to find a compound that could specifically target cyclin dependent kinases and cyclin complex inhibition.

\section{Acknowledgement}

The author wishes to acknowledge Dr. C. Swaminathan, Dr. M. Manivannan, Dr. S.Parial, all my teachers, as well as Modern Approaches in Drug Designing journal for their kindness.

\section{References}

1. Cao L, Chen F, Yang X, Xu W, Xie J, et al. (2014) Phylogenetic analysis of CDK and cyclin proteins in premetazoan lineages. BMC Evol Biol 14: 10.

2. Malumbres M, Barbacid M (2005) Mammalian cyclin-dependent kinases. Trends Biochem Sci 30(11): 630-641.

3. Boutros R, Lobjois V, Ducommun B (2007) CDC25 phosphatases in cancer cells: key players? Good targets? Nat Rev Cancer 7(7): 495-507.

4. (2006) Oncology: An Evidence-Based Approach. In: Chang AE, Ganz PA, Hayes DF, Kinsella T, Pass HI, et al. (Eds.), Oncology \& Hematology. Springer, New York, USA. 
5. YalakSHmi Sridhar, Nagaraan Pattabiraman, Eliot M Rosen, Richard G Pestell (2006) Inhibitors of Cyclin-dependent Kinases as Anti-tumor Agents. In: Smith PJ, Yue EW (Eds.), CDK inhibitors as anticancer agents: Pspetives for the future. Chap-18, CRC Press, Boca Raton, Florida, United States, p. 448.

6. Sedlacek H, Czech J, Naik R, Kaur G, Worland P, et al. (1996) Flavopiridol (L86 8275; NSC 649890), a new kinase inhibitor for tumor therapy. Int ] Oncol 9(6): 1143-1168.

7. Gadbois DM, Hamaguchi JR, Swank RA, Bradbury EM (1992) Staurosporine is a potent inhibitor of p34cdc2 and p34cdc2-like kinases. Biochem Biophys Res Commun 184(1): 80-85.

8. Neant I, Guerrier P (1988) 6-Dimethylaminopurine blocks starfish oocyte maturation by inhibiting a relevant protein kinase activity. Exp Cell Res 176(1): 68-79.

9. De Azevedo WF, Leclerc S, Meijer L, Havlicek L, Strnad M, et al. (1997) Inhibition of cyclin-dependent kinases by purine analogues: Crystal structure of human cdk2 complexed with roscovitine. Eur J Biochem 243(1-2): 518-526.

10. Akiyama T, Yoshida T, Tsujita T, Shimizu M, Mizukami T, et al. (1997) G1 phase accumulation induced by UCN-01 is associated with dephosphorylation of $\mathrm{Rb}$ and $\mathrm{CDK} 2$ proteins as well as induction of CDK inhibitor p21/Cip1/WAF1/Sdi1 in p53-mutated human epidermoid carcinoma A431 cells. Cancer Res 57(8): 1495-1501.

11. Malumbres M, Pevarello P, Barbacid M, Bischoff JR (2008) CDK inhibitors in cancer therapy: what is next? Trends Pharmacol Sci 29(1): 16-21.

12. Kitagawa M, Okabe T, Ogino H, Matsumoto H, Takahashi IS, et al. (1993) Butyrolactone I, a selective inhibitor of cdk2 and cdc2 kinase. Oncogene 8(9): 2425-2432.

13. Meijer L, Thunnissen AM, White A, Garnier M, Nikolic M, et al. (2000) Inhibition of cyclin-dependent kinases, GSK-3 $\beta$ and CK1 by Hymenial disine, a marine sponge constituent. Chem Biol 7(1): 51-63.

14. Soni R, Muller L, Furet P, Schoepfer J, Stephan C, et al. (2000) Inhibition of cyclin-dependent kinase $4(\mathrm{Cdk} 4)$ by fascaplysin, a marine natural product. Biochem Biophys Res Commun 275(3): 877-884.

15. Bettayeb K, Tirado OM, Lambot SM, Ferandin Y, Lozach O, et al. (2007) Meriolins, a new class of cell death inducing kinase inhibitors with enhanced selectivity for cyclin-dependent kinases. Cancer Res 67(17): 8325-8334.

16. Gompel M, Leost M, de Kier Joffe EB, Puricelli L, Franco LH, et al. (2004) Meridianins, a new family of protein kinase inhibitors isolated from the ascidian Aplidium meridianum. Bioorg Med Chem Lett 14(7): 1703-1707.

17. Perry NB, Ettouati L, Litaudon M, Blunt JW, Munro MHG, et al. (1994) Alkaloids from the Antarctic sponge Kirkpatrickia varialosa. Part1: Variolin B, a new antitumour and antiviral compound. Tetrahedron 50(13): 3987-3992.

18. Trimurtulu G, Faulkner DJ, Perry NB, Ettouati L, Litaudon M, et al. (1994) Alkaloids from the Antarctic sponge Kirkpatrickia varialosa. Part 2: Variolin A and N (3')-methyl tetrahydrovariolin B. Tetrahedron 50(13): 3993-4000.

19. Joshi KS, Rathos MJ, Joshi RD, Sivakumar M, Mascarenhas M, et al. (2007) In vitro antitumor properties of a novel cyclin-dependent kinase inhibitor, P276-00. Mol Cancer Ther 6(3): 918-925.
20. Joshi KS, Rathos MJ, Mahajan P, Wagh V, Shenoy S, et al. (2007) P27600, a novel cyclin-dependent inhibitor induces G1-G2 arrest, shows antitumor activity on cisplatin-resistant cells and significant in vivo efficacy in tumor models. Mol Cancer Ther 6(3): 926-934.

21. Misra RN, Xiao H, Kim KS, Lu S, Han WC, et al. (2004) N-(cycloalkylamino) acyl-2-aminothiazole inhibitors of cyclin-dependent kinase 2.N-[5-[[[5 - (1,1-dimethyle thyl) - 2-oxazolyl] methyl] thio]-2 thiazolyl]4Piperidinecarboxamide (BMS-387032), a highly efficacious and selective antitumor agent. J Med Chem 47(7): 1719-1728.

22. Kamath AV, Chong S Chang M, Marathe PH (2005) P-glycoprotein plays a role in the oral absorption of BMS-387032, a potent cyclin-dependent kinase 2 inhibitor, in rats. Cancer Chemother Pharmacol 55(2): 110-116.

23. Vassilev LT, Tovar C, Chen S, Knezevic D, Zhao X, et al. (2006) Selective small-molecule inhibitor reveals critical mitotic functions of human CDK1. Proc Natl Acad Sci U S A 103(28): 10660-10665.

24. Walsby E Lazenby M, Pepper C, Burnett AK (2011) The cyclin-dependent kinase inhibitor SNS-032 has single agent activity in AML cells and is highly synergistic with cytarabine. Leukemia 25(3): 411-419.

25. Kubo A, Nakagawa K, Varma RK, Conrad NK, Cheng JQ, et al. (1999) The p16 status of tumor cell lines identifies small molecule inhibitors specific for cyclin-dependent kinase 4. Clin Cancer Res 5(12): 4279-4286.

26. Diccianni MB, Yu J, Meppelink G, de Vries M, Shao L, et al. (2004) 3-amino thioacridone inhibits DNA synthesis and induce DNA damage in T-cell acute lymphoblastic leukemia (T-ALL) in a p16-dependent manner. J Exp Ther Oncol 4(3): 223-237.

27. Diccianni MB, Batova A, Yu J, Vu T, Pullen J, et al. (1997) Shortened survival after relapse in T-cell acute lymphoblastic leukemia patients with p16/p15 deletions. Leuk Res 21(6): 549-558.

28. Moshinsky DJ, Bellamacina CR, Boisvert DC, Huang P, Hui T, et al. (2003) SU9516: biochemical analysis of cdk inhibition and crystal structure in complex with cdk2. Biochem Biophys Res Commun 310(3): 1026-1031.

29. Lo M, Ngo R, Dai K (2005) Development of a time-resolved inhibitors of cyclin-dependent kinase/cyclin activation. J Biol Chem 14: 1379313800.

30. Canela N, Orzaez M, Fucho R, Mateo F, Gutierrez R, et al. (2006) Identification of an hexapeptide that binds to a surface pocket in cyclin a and inhibits the catalytic activity of the complex cyclin-dependent kinase 2-cyclin A. J Biol Chem 281(47): 35942-35953.

31. Bagella L, Sun A, Tonini T, Abbadessa G, Cottone G, et al. (2007) A small molecule based on the $\mathrm{pRb} 2 / \mathrm{p} 130$ spacer domain leads to inhibition of cdk2 activity, cell cycle arrest and tumor growth reduction in vivo. Oncogene 26(13): 1829-1839.

32. Liu S, Bolger JK, Kirkland LO, Premnath PN, McInnes C (2010) Structural and functional analysis of cyclin D1 reveals p27 and substrate inhibitor binding requirements. ACS Chem Biol 5(12): 1169-1182.

33. Martin MP, Alam R, Betzi S, Ingles DJ, Zhu JY, et al. (2012) A novel approach to the discovery of small molecule ligands of CDK2, Chembiochem 13(14): 2128-2136.

34. Liu H, Liu K, Huang Z, Park CM, Thimmegowda NR, et al. (2013) A chrysin derivative suppresses skin cancer growth by inhibiting cyclindependent kinases. J Biol Chem 288(36): 25924-25937. 